\title{
LIE AND JORDAN IDEALS OF OPERATORS ON HILBERT SPACE
}

\author{
C. K. FONG, C. R. MIERS AND A. R. SOUROUR
}

\begin{abstract}
A linear manifold $\mathfrak{Z}$ in $\mathfrak{B}(\mathfrak{B})$ is a Lie ideal in $\mathfrak{B}(\mathfrak{B})$ if and only if there is an associative ideal $\mathfrak{\Im}$ such that $[\mathfrak{J}, \mathfrak{B}(\mathfrak{Q})] \subseteq \mathfrak{L} \subseteq \mathfrak{I}+\mathbf{C I}$. Also $\mathfrak{L}$ is a Lie ideal if and only if it contains the unitary orbit of every operator in it. On the other hand, a subset of $\mathfrak{B}(\mathfrak{Q})$ is a Jordan ideal if and only if it is an associative ideal.
\end{abstract}

Let $\mathfrak{Q}$ be an infinite-dimensional separable Hilbert space and let $\mathfrak{B}(\mathfrak{Q})$ be the algebra of all bounded operators on $\mathfrak{Q}$. The algebra $\mathfrak{B}(\mathfrak{G})$ becomes a Lie algebra and a Jordan algebra under the products

$$
[A, B]=A B-B A \text { and } A \circ B=\frac{1}{2}(A B+B A)
$$

respectively. A linear manifold $\mathfrak{L}$ in $\mathfrak{B}(\mathfrak{S})$ is called a Lie ideal if $[A, X] \in \mathfrak{L}$ for every $A \in \mathfrak{B}(\mathfrak{Q})$ and $X \in \mathfrak{R}$. Jordan ideals are defined similarly. The purpose of this note is to investigate the relationship between the Lie ideals, the Jordan ideals and the associative ideals in $\mathfrak{B}(\mathfrak{E})$. (By an associative ideal, we mean a 2-sided ideal under the usual multiplication of operators.) Some of these results (as indicated below) were obtained by Topping in a manuscript [15] that was never published because of his untimely death. The results in Topping's manuscript were described to us by J. P. Williams but we were not able to locate the manuscript until after the first version of this paper was written. Topping's techniques were quite different from those we employ.

It should be noted that when $\mathscr{G}$ is finite dimensional, it is known that $\mathscr{B}(\mathfrak{G})$ contains no nontrivial associative or Jordan ideals and that the only nontrivial Lie ideals are the scalar multiples of the identity and the set of operators with trace zero. This can be proved directly and also follows from Chapter 1 of [7].

We employ standard notation and terminology. In particular, if $\mathfrak{\Im}$ is an ideal in an algebra $\mathfrak{B}$, then $[\mathfrak{\Im}, \mathfrak{B}$ ] denotes the set of all finite sums of elements of the form $A B-B A$ with $A \in \mathfrak{\Im}$ and $B \in \mathfrak{B}$. Linear manifolds are not assumed closed in any topology.

The implication $(2) \Rightarrow(1)$ in the following theorem was proved by Topping [14, proof of Theorem 1]. Topping knew the reverse implication, but his proof is not available to us; the proof for closed Lie ideals is in Topping [15].

Received by the editors November 10, 1980 and, in revised form, June 26, 1981.

1980 Mathematics Subject Classification. Primary 47D25. 
THEOREM 1. Let $\mathfrak{Q}$ be a linear manifold in $\mathfrak{B}(\mathfrak{Q})$. The following conditions are equivalent:

(1) $\mathbb{R}$ is a Lie ideal;

(2) $U^{*} \mathfrak{Q} U \subseteq \mathfrak{Q}$ for every unitary operator $U$;

(3) $T^{-1} \mathfrak{Q} T \subseteq \mathfrak{L}$ for every invertible operator $T$.

Proof. To prove the implication (1) $\Rightarrow(3)$, let $\mathfrak{\&}$ be a Lie ideal. A theorem of $W$. Gustafson, P. R. Halmos and H. Radjavi (see [9]) asserts that every invertible operator is the product of seven (oblique) symmetries, i.e. square roots of the identity. So it suffices to show that $\mathfrak{R}$ is closed under conjugation by symmetries. If $S$ is a symmetry and $A \in \mathfrak{R}$, then $S^{-1} A S=A-\frac{1}{2}[S,[S, A]] \in \mathfrak{R}$.

The implication $(3) \Rightarrow(2)$ is obvious and the implication $(2) \Rightarrow(1)$ is contained in [14, proof of Theorem 1]; the short proof is given here for completeness. Assume that $\mathfrak{L}$ satisfies condition (2). By a theorem of Fillmore [4], every operator is a linear combination of a finite number of projections, so it suffices to show that $[P, \mathfrak{R}] \subseteq \mathfrak{L}$ for every projection $P$. If $P$ is a projection and $A \in \mathfrak{L}$, then $U=P+i(1-P)$ is unitary and $[P, A]=\left(U^{*} A U-U A U^{*}\right) / 2 i \in \mathfrak{R}$.

Before stating our main result, we remind the reader of certain definitions and observations concerning ideals of operators on a Hilbert space. (Ideals are two-sided associative ideals, and Hilbert spaces are infinite dimensional and separable.) If $T$ is a compact operator from a Hilbert space $\Omega_{1}$ into a Hilbert space $\Omega_{2}$, then the sequence of $s$-numbers of $T$ is the decreasing sequence of nonzero eigenvalues of the compact positive operator $\left(T^{*} T\right)^{1 / 2}$, each repeated according to its multiplicity. There is a one-to-one correspondence between certain sets of sequences, called ideal sets, and ideals in $\mathfrak{B}(\mathfrak{E})$ [10, pp. 25-27]. The relationship between operator ideals and ideal sets is as follows: a compact operator $T: \mathfrak{B} \rightarrow \mathfrak{G}$ belongs to an ideal $\mathfrak{\Im} \subseteq \mathfrak{B}(\mathfrak{Q})$ if and only if its sequence of $s$-numbers belongs to the corresponding ideal set. Following [2] we say that a compact operator $T: \Re_{1} \rightarrow \Re_{2}$ is affiliated with the ideal $\mathfrak{s} \subseteq \mathfrak{B}(\mathfrak{Q})$ if the sequence of $s$-numbers of $T$ belongs to the ideal set corresponding to $\mathfrak{\Im}$. We omit the easy proof of the following lemma.

LemMA. (a) The operator $T: \Re_{1} \rightarrow \Re_{2}$ is affiliated with the ideal $\mathfrak{\Im} \subseteq \mathfrak{B}(\mathfrak{S})$ if and only if $W^{*} T V \in \mathfrak{\Im}$ for some pair of unitary operators $V$ and $W$ of $\mathfrak{Q}$ onto $\Omega_{1}$ and $\Omega_{2}$ respectively, and this is true if and only if $W^{*} T V \in \mathfrak{\Im}$ for every such pair of unitaries $V$ and $W$.

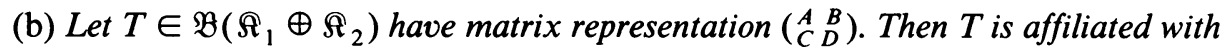
$\mathfrak{\Im} \subseteq \mathfrak{B}(\mathfrak{G})$ if and only if $A, B, C, D$ are affiliated with $\mathfrak{J}$.

Let $\mathfrak{R}$ be a Lie ideal in $\mathfrak{B}(\mathfrak{Q})$, let $\mathfrak{A}$ be another Hilbert space, and let $\tilde{\mathfrak{L}}$ be the set of operators on $\mathfrak{\Omega}$ which are unitarily equivalent to the operators in $\mathfrak{L}$. In view of Theorem $1, \tilde{\mathfrak{L}}$ is a Lie ideal in $\mathfrak{B}(\mathfrak{\Omega})$.

THEOREM 2. Let $\mathfrak{Z}$ be a Lie ideal in $\mathfrak{B}(\mathfrak{Q})$. Then there is an associative ideal $\mathfrak{\Im}$ such that

$$
[\mathfrak{\Im}, \mathfrak{B}(\mathfrak{Q})] \subseteq \mathfrak{L} \subseteq \mathfrak{\Im}+\mathbf{C} 1 .
$$

Conversely, if $\mathfrak{\Im}$ is an associative ideal and $\mathfrak{\Omega}$ is a linear space satisfying the inclusions $(\dagger)$, then $\mathfrak{Z}$ is a Lie ideal in $\mathfrak{B}(\mathfrak{Q})$. 
Proof. Assume that $\mathfrak{L}$ is a Lie ideal in $\mathfrak{B}(\mathfrak{G})$ and let $\tilde{\mathfrak{L}}$ be the corresponding Lie ideal in $\mathfrak{B}(\mathfrak{G} \oplus \mathfrak{G})$. Let

$$
\mathfrak{\Im}=\left\{B \in \mathfrak{B}(\mathfrak{Q}):\left(\begin{array}{ll}
0 & B \\
0 & 0
\end{array}\right) \in \tilde{\mathfrak{I}}\right\} .
$$

We now show that $\mathfrak{\Im}$ is a two-sided ideal in $\mathfrak{B}(\mathfrak{F})$. It is immediate that $\mathfrak{\Im}$ is a linear space. Furthermore if $B \in \mathfrak{\Im}$ and $T \in \mathfrak{B}(\mathfrak{Q})$, then

$$
\left(\begin{array}{cc}
0 & T B \\
0 & 0
\end{array}\right)=\left[\left(\begin{array}{ll}
T & 0 \\
0 & 0
\end{array}\right),\left(\begin{array}{ll}
0 & B \\
0 & 0
\end{array}\right)\right] \in \tilde{\mathfrak{L}}
$$

and

$$
\left(\begin{array}{cc}
0 & B T \\
0 & 0
\end{array}\right)=\left[\left(\begin{array}{cc}
0 & B \\
0 & 0
\end{array}\right),\left(\begin{array}{ll}
0 & 0 \\
0 & T
\end{array}\right)\right] \in \tilde{\mathfrak{L}}
$$

so $B T$ and $T B \in \mathfrak{\Im}$.

To show that $\mathfrak{L} \subseteq \mathfrak{\Im}+\mathbf{C}$, take $T \in \mathfrak{L}$. Let $P$ be a projection with infinitedimensional range $\mathfrak{M}$ and infinite-dimensional null space $\mathfrak{R}$. We will show that $T P-P T \in \mathfrak{S}$. Relative to the decomposition $\mathfrak{Q}=\mathfrak{M} \oplus \mathfrak{R}$, the operator $P$ has the matrix $\left(\begin{array}{ll}1 & 0 \\ 0 & 0\end{array}\right)$. Assume that the matrix of $T$ relative to the same decomposition is $\left(\begin{array}{ll}A & B \\ C & D\end{array}\right)$. Therefore

$$
[T, P]=\left(\begin{array}{cc}
0 & -B \\
C & 0
\end{array}\right) \in \mathfrak{\Omega}
$$

Furthermore

$$
[[T, P], P]=\left(\begin{array}{ll}
0 & B \\
C & 0
\end{array}\right) \in \mathfrak{R},
$$

so $\left(\begin{array}{ll}0 & B \\ 0 & 0\end{array}\right)$ belongs to $\mathfrak{R}$. If $V$ and $W$ are unitary maps of $\mathfrak{B}$ onto $\mathfrak{M}$ and $\mathfrak{N}$ respectively, then the operator

$$
\left(\begin{array}{cc}
0 & W^{*} B V \\
0 & 0
\end{array}\right) \text { on } \mathfrak{G} \oplus \mathfrak{Q}
$$

belongs to $\tilde{\mathfrak{L}}$ and so $W^{*} B V \in \mathfrak{\Im}$. By the lemma, the operator $B$ is affiliated with $\mathfrak{\Im}$ and hence $\left(\begin{array}{ll}0 & B \\ 0 & 0\end{array}\right)$ must also be affiliated with $\mathfrak{J}$. But $\left(\begin{array}{ll}0 & B \\ 0 & 0\end{array}\right)$ is an operator on $\mathfrak{G}$ and so must belong to $\mathfrak{\Im}$. Similarly $\left(\begin{array}{cc}0 & 0 \\ C & 0\end{array}\right)$ also belongs to $\mathfrak{\Im}$ and hence

$$
[T, P]=\left(\begin{array}{cc}
0 & -B \\
C & 0
\end{array}\right) \in \mathfrak{\Im}
$$

By taking sums and differences of projections, we obtain that $[T, P] \in \mathfrak{\Im}$ for every projection $P$ regardless of its rank or its corank. By Fillmore's theorem [4], we obtain that $[T, S] \in \mathfrak{\Im}$ for every $S$ in $\mathfrak{B}(\mathfrak{Q})$. A theorem of Calkin [3] asserts that the last condition implies that $T \in \mathfrak{\Im}+\mathbf{C} 1$.

Next we show that $[\mathfrak{\Im}, \mathfrak{B}(\mathfrak{Q})] \subseteq \mathfrak{L}$. As before it is enough to show that $[B, P] \subset \mathfrak{Z}$ for every $B \in \mathfrak{\Im}$ and every projection $P$ of infinite rank and infinite corank. Write

$$
P=\left(\begin{array}{ll}
1 & 0 \\
0 & 0
\end{array}\right) \text { and } B=\left(\begin{array}{ll}
R & S \\
T & U
\end{array}\right)
$$


The operators $R, S, T, U$ are affiliated with $\mathfrak{\Im}$. So $\left(\begin{array}{ll}0 & S \\ 0 & 0\end{array}\right)$ and $\left(\begin{array}{ll}0 & 0 \\ T & 0\end{array}\right)$ belong to $\mathfrak{\Im}$. Therefore

$$
[B, P]=\left(\begin{array}{cc}
0 & -S \\
T & 0
\end{array}\right) \in \mathfrak{\Im} .
$$

This ends the proof of the first assertion of the theorem. The converse is obvious.

COROLlaRY 1 (TOPPING). If $\mathfrak{Q}$ is a Lie ideal in $\mathfrak{B}(\mathfrak{Q})$, then either $\mathfrak{L}=\mathfrak{B}(\mathfrak{B})$ or $\mathfrak{L} \subseteq \Re(\mathfrak{Q})+\mathbf{C l}$, where $\mathfrak{\Omega}(\mathfrak{Q})$ is the ideal of compact operators.

Proof. Let $\mathfrak{s}$ be an associative ideal related to $\mathfrak{L}$ as in Theorem 2 . If $\mathfrak{\Im}=\mathfrak{B}(\mathfrak{Q})$, then by $[5$, Problem 186] we have $[\mathfrak{\Im}, \mathfrak{B}(\mathfrak{Q})]=\mathfrak{B}(\mathfrak{Q})$ and $\mathfrak{L}=\mathfrak{B}(\mathfrak{Q})$. Otherwise, $\mathfrak{\Im} \subseteq \Re(\mathfrak{Q})$ and $\mathfrak{L} \subseteq \mathfrak{\Im}+\mathbf{C} 1 \subseteq \Re(\mathfrak{Q})+\mathbf{C l}$.

The following corollary is a special case of a result of Topping [14] and also follows from [7, Theorem 1.3] and [5, Problem 186].

COROllary 2 (TOPPING). The set of scalars $\mathrm{C} 1$ is the only nontrivial Lie ideal in the Calkin algebra $\mathfrak{B}(\mathfrak{S}) / \mathfrak{R}(\mathfrak{B})$.

Proof. Let $\pi: \mathfrak{B}(\mathfrak{E}) \rightarrow \mathfrak{A}$ be the natural projection of $\mathfrak{B}(\mathfrak{E})$ onto the Calkin algebra $\mathfrak{A}$. If $\mathfrak{L}$ is a Lie ideal in $\mathfrak{A}$, then $\pi^{-1}(\mathfrak{L})$ is a Lie ideal in $\mathfrak{B}(\mathfrak{S})$ containing $\mathfrak{\Omega}(\mathfrak{Q})$. So $\pi^{-1}(\mathfrak{L})=\mathfrak{B}(\mathfrak{Q})$ or $\mathfrak{A}(\mathfrak{Q})+\mathbf{C l}$ or $\mathfrak{R}(\mathfrak{Q})$. Therefore $\mathfrak{L}$ is either $\mathfrak{A}$ or $\mathbf{C l}$ or $\{0\}$.

COROLlARY 3 (TOPPING). There are precisely three nontrivial closed Lie ideals in $\mathfrak{B}(\mathfrak{\mathcal { Q }})$, namely $\mathrm{Cl}, \mathfrak{\Re}(\mathfrak{S})$ and $\mathfrak{\Re}(\mathfrak{Q})+\mathbf{C l}$.

Proof. Let $\mathfrak{R}$ be a nontrivial closed Lie ideal in $\mathfrak{B}(\mathfrak{S})$ and let $\mathfrak{s}$ be an associative ideal related to $\mathfrak{L}$ as in Theorem 2 . If $\mathfrak{s}=\{0\}$, then $\mathfrak{L} \subseteq \mathbf{C 1}$ and so $\mathfrak{L}=\mathbf{C 1}$. If $\mathfrak{J} \neq\{0\}$, then $\mathfrak{\Im}$ contains the ideal of finite rank operators $\mathfrak{F}$ and $\mathfrak{L}$ contains $[\mathfrak{F}, \mathfrak{B}(\mathfrak{E})]$. But $[\mathfrak{F}, \mathfrak{B}(\mathfrak{Q})]=\mathfrak{F}^{0}$, the set of finite rank operators with trace zero. (The last assertion can be easily reduced to the finite-dimensional case [11].) Furthermore $\mathfrak{F}^{0}$ is dense in $\mathscr{\Re}(\mathfrak{S})$ since $\mathfrak{F}$ is dense in $\mathscr{R}(\mathfrak{Q})$, and for any $F \in \mathfrak{F}$ with trace $t$, the sequence $\left\{F-(t / n) P_{n}\right\}$, where $P_{n}$ are projections of rank $n$, converges to $F$ and consists of operators in $\mathfrak{F}^{0}$. So $\mathfrak{L} \supseteq \mathfrak{I}(\mathfrak{E})$ and, by Lemma $1, \mathfrak{L}$ is either $\mathscr{\Re}(\mathfrak{E})$ or $\mathfrak{\Omega}(\mathfrak{G})+\mathbf{C l}$.

REMARKs. 1. Theorem 2 raises the question of identifying the commutator subspaces $[\mathfrak{\Im}, \mathfrak{B}(\mathfrak{\mathcal { Q }})]$. For certain ideals $\mathfrak{\Im}$, it is known that $[\mathfrak{\Im}, \mathfrak{B}(\mathfrak{Y})]=\mathfrak{J}$. This is the case when $\mathfrak{\Im}=\mathscr{\Re}(\mathfrak{S})$ (Pearcy and Topping [8], see also Anderson [1]) and when $\mathfrak{s}=\mathfrak{S}_{p}, p>1$ (this can be proved by modification of the proofs in Pearcy and Topping [8]). But if $\mathfrak{F}$ is the ideal of finite rank operators, then $[\mathfrak{F}, \mathfrak{B}(\mathfrak{Q})]=\mathfrak{F}^{0}$, the class of operators in $\mathfrak{F}$ with trace zero. Furthermore, $\left[\mathfrak{F}_{1}, \mathfrak{B}(\mathfrak{S})\right]$ is properly contained in $\mathfrak{S}_{1}^{0}$, the set of operators with trace zero. To prove this let $T \in \mathfrak{B}(\mathfrak{S})$ and $C \in \mathfrak{E}_{1}$. Then $C=A B$ with $A$ and $B \in \mathfrak{E}_{2}$. So $[T, C]=[T A, B]+[B T, A] \in$ $\left[\mathfrak{E}_{2}, \mathbb{E}_{2}\right]$ which was shown by Weiss $[16]$ to be properly contained in $\mathfrak{\mho}_{1}^{0}$.

2. In [12, Lemma 2], it was shown that if $\mathfrak{L}$ is a linear manifold of compact operators closed under unitary equivalence (by Theorem 1, this is equivalent to assuming that $\mathfrak{Z}$ is a Lie ideal of compact operators), then under the extra 
assumptions that $\mathfrak{Z}$ is closed under compression and that it contains the HilbertSchmidt operators, it follows that $\mathfrak{R}$ is an associative ideal. The proof in [12] can be easily modified to prove this when $\mathfrak{Q}$ contains the trace class operators instead of the Hilbert-Schmidt operators. We do not know if the result is true without assuming that $\mathfrak{R}$ is closed under compression.

3. The Lie ideals in $\mathfrak{L}(\mathfrak{B})$, the algebra of all linear transformations on an infinite-dimensional vector space $\mathfrak{B}$ (with no topology), have been characterized by Stewart [13].

We now turn our attention to the Jordan ideals.

THEOREM 3. A subset of $\mathfrak{B}(\mathfrak{Q})$ is a Jordan ideal if and only if it is an associative ideal.

Proof. The "if" part is trivial. To prove the "only if" part let $\mathfrak{\Im}$ be a Jordan ideal and let $T \in \mathfrak{\Im}$. It suffices to show that $T P$ and $P T \in \mathfrak{\Im}$ for an arbitrary projection $P$ of infinite rank and infinite corank. First we note that $P T P=2(T \circ P) \circ P-T \circ P$ and so $P T P \in \mathfrak{\Im}$. Let $V$ be a partial isometry such that $V V^{*}=P$ and $V^{*} V=(1-P)$. We have $T \circ V \in \mathfrak{S}$ and so $P(T \circ V) P \in \mathfrak{\Im}$. Using $V P=0$ and $P V=V$, we obtain $V T P \in \mathfrak{\Im}$. Therefore $(V T P) \circ V^{*} \in \mathfrak{\Im}$. Using $P V^{*}=0$, we get $(1-P) T P \in \mathfrak{\Im}$. This, together with $P T P \in \mathfrak{\Im}$, imply that $T P \in \mathfrak{\Im}$. We must also have $P T=2 T \circ P$ $-T P \in \mathfrak{s}$.

\section{REFERENCES}

1. J. Anderson, Commutators of compact operators, J. Reine Angew. Math. 291 (1977), 128-132.

2. A. Brown and C. Pearcy, Compact restrictions of operators, Acta Sci. Math. 32 (1971), 271-282.

3. J. W. Calkin, Two-sided ideals and congruences in the ring of bounded operators in Hilbert space, Ann. of Math. (2) 42 (1941), 839-873.

4. P. Fillmore, Sums of operators with square zero, Acta Sci. Math. 28 (1967), 285-288.

5. P. R. Halmos, A Hilbert space problem book, Van Nostrand, New York, 1967.

6. P. R. Halmos and S. Kakutani, Products of symmetries, Bull. Amer. Math. Soc. 64 (1958), 77-78.

7. I. N. Herstein, Topics in ring theory, Univ. of Chicago Press, Chicago, Ill., 1969.

8. C. Pearcy and D. Topping, On commutators in ideals of compact operators, Michigan Math. J. 18 (1971), 247-252.

9. H. Radjavi, The group generated by involutions, Proc. Roy. Irish Acad. Sect. A 81 (1981).

10. R. Schatten, Norm ideals of completely continuous operators, Springer-Verlag, Berlin, 1960.

11. K. Shoda, Einige Sätze über Matrizen, Japan. J. Math. 13 (1936), 261-265.

12. A. R. Sourour, A note on integral operators, Acta Sci. Math. 41 (1979), 375-379.

13. I. Stewart, The Lie algebra of endomorphisms of an infinite-dimensional vector space, Compositio Math. 25 (1972), 79-86.

14. D. Topping, On linear combinations of special operators, J. Algebra 10 (1968), 516-521.

15.

16. G. Weiss, Commutators of Hilbert-Schmidt operators, preprint.

Department of Mathematics, University of Toronto, Toronto, Ontario, M5S 1A1, Canada (Current address of C. K. Fong)

Department of Mathematics, University of Victoria, Victoria, British Columbia V8W 2Y2, Canada (Current address of C. R. Miers and A. R. Sourour) 